\title{
CENTRAL LACUNARY SETS FOR LIE GROUPS
}

\author{
A. H. DOOLEY
}

(Received 9 October 1986)

Communicated by J. F. Price

Dedicated to Robert Edwards in recognition of 25 years' distinguished contribution to mathematics in Australia on the occasion of his retirement

\begin{abstract}
If $G$ is a compact connected Lie group every infinite subset of $\hat{G}$ contains an infinite central $\Lambda(p)$ set, for $p<2+2 \operatorname{rank} G /(\operatorname{dim} G-\operatorname{rank} G)$. A subset $R$ of $\hat{G}$ is of type central $\Lambda(2)$ if and only if the associated set of characters on the maximal torus is of type $\Lambda(2)$. The dual of a compact connected semisimple Lie group contains infinite sets which are central $p$-Sidon for all $p>1$. Every infinite subset of the dual of $S U(2)$ contains such a set.
\end{abstract}

1980 Mathematics subject classification (Amer. Math. Soc.): 42 A 44, 43 A 14.

\section{Introduction}

The notion of $p$-Sidon set $(1<p<2)$ was introduced for compact abelian groups by Edwards and Ross [5]. These authors showed that there exist $\frac{4}{3}$-Sidon sets which are not Sidon sets. Their construction was elaborated by Johnson and Woodward [7] who found $2 n /(2 n-1)$-Sidon sets which are not $(2 n-1) /(2 n-3)$ Sidon sets. Recently, interest in this problem has resurfaced with work of $R$. Blei [1], who uses techniques of combinatorial dimension to show that for all $1 \leq p_{1}<p_{2}<2$ there exist $p_{2}$-Sidon sets which are not $p_{1}$-Sidon.

It is known that for compact connected nonabelian groups the theory of lacunarity departs significantly from the abelian theory. For ecample, a compact, connected semisimple Lie group has no infinite Sidon sets, infinite central Sidon

(C) 1988 Australian Mathematical Society $0263-6115 / 88 \$ A 2.00+0.00$ 
sets, or infinite $p$-Sidon sets (see [4], [8], [10]). Cartwright and McMullen have recently characterized those compact connected groups which have infinite Sidon sets.

However, there is one perplexing gap in these results, the question of whether a compact connected semisimple Lie group can have infinite central $p$-Sidon sets. The major part of this paper (§4) answers this question in the affirmative. In fact, every compact connected semisimple Lie group has an infinite set in its dual which is central $p$-Sidon for all $p>1$ (but no infinite central Sidon (= central 1-Sidon) sets). For $S U(2)$, one can say more; every infinite subset of the dual contains an infinite set which is central $p$-Sidon for every $p>1$.

This result is proved in the context of sets of type central $\Lambda(p, q)$ and central $V(p, q)$ previously discussed by the author [3]. (Note that $V\left(1, p^{\prime}\right)=p$-Sidon.) A few ancillary results are obtained from these sets, which enable one to fill some gaps left in [3], showing in particular that these classes, too, are strictly larger than previously considered classes. (There are sets of type central $V(s, r)$ which are not central $\Lambda(2)$.)

Preliminary notions from harmonic analysis and Lie theory are set forth in §1. In an effort to make the article more accessible to harmonic analysts who are not specialists in Lie theory, I have included a reasonably complete summary of requisites from this area.

\section{Preliminaries}

(1.1) Let $G$ be a compact (Hausdorff) topological group, which normalized Haar measure $\lambda_{G}$. If $f$ is a continuous function on $G$, define as usual $\|f\|_{p}=$ $\left(\int|f|^{p} d \lambda_{G}\right)^{1 / p}$. If $f$ and $g$ are both continuous functions, their convolution is $f * g(x)=\int f\left(x y^{-1}\right) g(y) d \lambda_{G}(y)$. By $\hat{G}, I$ denote a maximal set of pairwise inequivalent irreducible representations of $G$; each $\sigma \in \hat{G}$ acts in a Hilbert space $\mathscr{H}_{\sigma}$ of dimension $d_{\sigma}$, and $\chi_{\sigma}$ denotes the character $x \mapsto \operatorname{tr} \sigma(x)$ of $\sigma$. If $f$ belongs to the convolution centre of the space of continuous functions, its Fourier series is

$$
f \sim \sum d_{\sigma} a_{\sigma} \chi_{\sigma}, \quad \text { where } a_{\sigma}=\frac{1}{d_{\sigma}} \int f \cdot \bar{\chi}_{\sigma} d \lambda_{G} .
$$

A subset of $R$ of $\hat{G}$ is said to be of type central $\Lambda(p, r)$ if there exists $K_{1} \in \mathbf{R}$ so that for all sequences $\left(a_{\sigma}\right)_{\sigma \in R}$ with $a_{\sigma}=0$ off a finite set,

$$
\left\|\sum_{\sigma \in R} a_{\sigma} \chi_{\sigma}\right\|_{p} \leq K_{1}\left(\sum_{\sigma \in R} d_{\sigma}^{2-r}\left|a_{\sigma}\right|^{r}\right)^{1 / r},
$$


and of type central $V(p, r)$ if there exists $K_{2} \in \mathbf{R}$ so that for all such sequences $\left(a_{\sigma}\right)_{\sigma \in R}$

$$
\left(\sum d_{\sigma}^{2-r^{\prime}}\left|a_{\sigma}\right|^{r^{\prime}}\right)^{1 / r^{\prime}} \leq K_{2}\left\|\sum_{\sigma \in R} a_{\sigma} \chi_{\sigma}\right\|_{p^{\prime}} .
$$

These sets have been discussed by the author in [2], [3].

(1.2) Let $G$ be a compact connected Lie group of rank $l$, and let $T=T^{l}$ be a maximal torus for $G$. The Weyl group $W$ is the finite group $N_{G}(T) / T$, where $N_{G}(T)$ denotes the normalizer of $T$ in $G$, and has an action on $T$ given by $w=g T: t \rightarrow w t=g t g^{-1}$. For each $w \in W$, sgn $w$ denotes the sign of the determinant of the derivative map $w_{*, e}$ (which is a map $\mathbf{R}^{l} \rightarrow \mathbf{R}^{l}$ ). Let $\Phi$ denote the set of roots of $G$ on $T: \Phi$ is the set of characters of $T$ which occur in the decomposition of the restriction to $T$ of the adjoint representation of $G$. Choose a base $\Delta$ for $\Phi$, that is, a set of $l$ linearly independent characters such that each element of $\Phi$ is $\prod_{\beta \in \Delta} \beta^{k_{\beta}}$, with the $k_{\beta}$ all integers of the same sign or zero, and denote by $\Phi^{+}$the set of positive roots (those with all $k_{\beta}$ 's nonnegative). The centre of $G$ is contained in $T$ and is precisely $\{x \in T \mid \alpha(x)=1$ for all $\alpha \in \Phi\}$. Note that each $\alpha \in \Phi$ defines a reflection $w_{\alpha} \in W$ and that these reflections generate $W$. Further, define a partial order $\prec$ on $\hat{T}$ (the set of characters of $T$ ) by $\chi_{1} \prec X_{2}$ if $\chi_{1} \bar{\chi}_{2}=\prod_{\beta \in \Delta} \beta^{k_{\beta}}$ with $k_{\beta}$ non-negative integers.

Now for each $\sigma \in \hat{G}, \chi_{\sigma} i r$ is a sum of characters and we write $\chi_{\sigma} i_{T}=$ $\sum_{\chi \in \hat{T}} n_{\sigma}(\chi) \cdot \chi$. It can be shown that there is, in this decomposition, just one character $\chi^{(\sigma)}$ which is maximal with respect to $\prec$, and that this character occurs precisely once. The representation $\sigma$ is said to be of highest weight $\chi^{(\sigma)}$, and the set $\left\{\chi^{(\sigma)}: \sigma \in \hat{G}\right\}$ of dominant weights is denoted $\hat{T}^{+}$. The map $\sigma \mapsto \chi^{(\sigma)}$ sets up a bijection $\hat{G} \rightarrow \hat{T}^{+}$. If $R \subseteq \hat{G}$, let $X(R)=\left\{\chi^{(\sigma)}: \sigma \in R\right\} \subseteq \hat{T}^{+}$. Elements of $W$ have a naturally defined action on $\hat{T}$-under this action, each character is conjugate to just one dominant weight. For $w \in W, \chi_{(w)}=\left(\prod_{\alpha \in \Phi^{+}} \alpha \cdot \overline{w \alpha}\right)^{1 / 2}$ is a character of $T$.

I shall need two formulae: the Weyl integration formula states that if $f$ is a continuous function on $G$,

$$
\int f d \lambda_{G}=\frac{1}{\operatorname{card} W} \int\left(\int f\left(x t x^{-1}\right) d \lambda_{G}(x)\right)|q(t)|^{2} d \lambda_{T}(t)
$$

where $q=\prod_{\alpha \in \Phi+}\left(1-\chi_{\alpha}\right)=\sum_{w \in W} \operatorname{sgn} w \bar{\chi}_{(w)}$, and the Weyl character formula that

$$
q \cdot \chi_{\sigma} \uparrow_{T}=\sum_{w \in W} \operatorname{sgn} w\left(w \chi^{(\sigma)}\right) \cdot \bar{\chi}_{(w)} .
$$

(1.3) Suppose in addition that $G$ is semisimple. For every $\chi \in \hat{T}$, the derivative map $\chi_{*, e}: \mathbb{R} \rightarrow \mathbb{R}$ is a homomorphism; let $\tilde{\chi}$ denote the unique extension of $\chi_{*, e}$ to a complex homomorphism $\mathbb{C}^{l} \rightarrow \mathbb{C}$. The elements $\tilde{\chi}, \chi \in \hat{T}$ are called the 
weights of $G$. Since $G$ is semisimple, the Killing form $\kappa(X, Y)=\operatorname{tr}(\operatorname{ad} X \circ$ ad $Y)$ defines a non-degenerate bilinear form on the complexified Lie algebra of $G$, whose restriction to the complexified Lie algebra of $T$ (that is, $\mathbb{C}^{l}$ ) gives it the structure of Euclidean space.

We may use duality to transfer $\kappa \int^{l} \times \mathbf{C}^{l}$ to a bilinear form $($,$) on \mathbb{C}^{l^{*}} \times \mathbb{C}^{l^{*}}$ : we then set

$$
\langle X, Y\rangle=\frac{2(X, Y)}{(Y, Y)} \quad \text { for } X, Y \in \mathbb{C}^{l^{*}}
$$

The Weyl dimension formula [15] states that

$$
d_{\sigma}=\prod_{\alpha \in \Phi^{+}} \frac{\left\langle\tilde{\chi}^{(\sigma)}+\tilde{\rho}, \alpha\right\rangle}{\langle\tilde{\rho}, \tilde{\alpha}\rangle}
$$

where $\tilde{\rho}=\frac{1}{2} \sum_{\alpha \in \Phi^{+}} \tilde{\alpha}$.

We can choose a basis $\left\{\tilde{\lambda}_{\beta}: \beta \in \Delta\right\}$ for $\left(\mathbb{C}^{l}\right)^{*}$ so that for all $\alpha, \beta \in \Delta,\left\langle\tilde{\lambda}_{\beta}, \tilde{\alpha}\right\rangle=$ $\delta_{\alpha, \beta}$. We then have $\tilde{\rho}=\sum_{\beta \in \Delta} \tilde{\lambda}_{\beta}$. If $\tilde{\chi}$ is a weight of $G$, then $\chi \in \hat{T}^{+}$if and only if $\tilde{\chi}=\sum_{\beta \in \Delta} m_{\beta} \tilde{\lambda}_{\beta}$ where the $m_{\beta}$ 's are nonnegative integers. If $G$ is simply connected, then every element of the form $\sum_{\beta \in \Delta} m_{\beta} \tilde{\lambda}_{\beta}$ with $m_{\beta} \in \mathbf{Z}^{+}$is an element of $\hat{T}^{+\sim}$.

Suppose $\tilde{\rho}$ is a weight of $G$. (This is not always true, but it is true if $G$ is simply connected; $2 \tilde{\rho}$ is always a weight of $G$.) Denote by $\rho \in \hat{T}^{+}$the associated character. Then $\chi_{(w)}=\rho \overline{w \rho}(w \in W)$, and we may write the Weyl character formula in its more usual form $\left.\chi_{\sigma}\right|_{T}=\mathcal{A}\left(\chi^{(\sigma)} \rho\right) / \mathcal{A}(\rho)$, where for $\chi \in \hat{T}, \mathcal{A}(\chi)=$ $\sum_{w \in W} \operatorname{sgn} w w(\chi)$.

(1.4) I shall always denote by $\varepsilon_{G}$ the number $l / \operatorname{card} \Phi^{+}$; since $\operatorname{dim} G=$ 2 card $\Phi^{+}+l$, and $l=\operatorname{rank} G$, we have $\varepsilon_{G}=2 \operatorname{rank} G /(\operatorname{dim} G-\operatorname{rank} G)$. It is known (see [3], Theorem 2.2) that if $s<\varepsilon_{G}$, then $\int|q|^{-s} d \lambda_{T}<\infty$.

(1.5) It will be convenient to define the notion of dimension $d(\chi)$ of an arbitrary character $\chi \in \hat{T}$. To this end, notice that $\tilde{\chi}+\tilde{\rho}$ is a weight of the complexified Lie algebra of $G$ (that is, has the form $\sum_{\beta \in \Delta} m_{\beta} \tilde{\lambda}_{\beta}$ with $m_{\beta} \in \mathbb{Z}$ ). Thus ([6], §13.2, Lemma A), there is an element $w$ of $W$ so that $\tilde{\lambda}=w(\tilde{\chi}+\tilde{\rho})$ is a dominant weight (that is, $\tilde{\lambda}=\sum_{\beta \in \Delta} k_{\beta} \tilde{\lambda}_{\beta}, k_{\beta} \geq 0$ ). If $\chi$ is such that $\tilde{\lambda}$ is strongly dominant $\left(k_{\beta}>0\right)$, then the choice of $w$ is unique, and $\tilde{\mu}=\tilde{\lambda}-\tilde{\rho}$ is again dominant; in fact, it is the differential of the character $w(\chi) \bar{\chi}_{(w)}$ of $T$. Thus $\tilde{\mu}$ corresponds to a representation $\sigma$ of $G$ and we define $d(\chi)=d_{\sigma}$. In the case where $\tilde{\lambda}$ is not strongly dominant, let $d(\chi)=0$. Notice $\hat{T}=\bigcup_{w \in W} w\left(\hat{T}^{+}\right) \bar{\chi}_{(w)}$, that $d$ is zero on the intersection $w\left(\hat{T}^{+}\right) \bar{\chi}_{(w)} \cap w^{\prime}\left(\hat{T}^{+}\right) \bar{\chi}_{\left(w^{\prime}\right)}, w \neq w^{\prime}$. 


\section{Sets of type central $\Lambda(p, q)$}

(2.1) Definition. Suppose $T$ is a compact abelian group and suppose $w: \hat{T} \rightarrow$ $\mathbf{R}^{+} \cup\{0\}$. Further suppose $p, s \in[1, \infty], s \neq \infty$. Then $Y \subseteq \hat{T}$ is said to be of type $w-\Lambda(p, s)$ it there exists $K \in \mathbf{R}$ such that for all sequences $\left\{a_{\chi}\right\}_{X \in Y}$, zero off a finite set,

$$
\left\|\sum_{\chi \in Y} a_{\chi} \cdot \chi\right\|_{p} \leq K\left(\sum_{\chi \in Y} w(\chi)\left|a_{\chi}\right|^{s}\right)^{1 / s} .
$$

These sets are related to the weighted lacunary sets of [14]. I shall only be interested in the case where $T$ is the maximal torus for $G, Y \subseteq \hat{T}^{+}$, and $w$ is $d^{r}$ (where $d$ is introduced in (1.5)).

(2.2) THEOREM. Suppose $G$ is a compact connected Lie group, $T$ a maximal torus for $G$. Let $R \subseteq \hat{G}$ and $r \in\left(2,2+\varepsilon_{G}\right), s \in[1, \infty]$. In order that $R$ should be of type central $\Lambda(r, s)$, it is sufficient that $X(R) \subseteq \hat{T}^{+}$should be of type $d^{2-s}-\Lambda(p, s)$ for some $p \in\left(\left(r \cdot \varepsilon_{G}\right) /\left(2+\varepsilon_{G}-r\right), \infty\right)$.

Proof. Choose $p \in\left(\left(r \cdot \varepsilon_{G}\right) /\left(2+\varepsilon_{G}-r\right), \infty\right)$. Note that since $2<r<2+\varepsilon_{G}$, we have $1<\varepsilon_{G} /\left(2+\varepsilon_{G}-r\right)<p / r<\infty$. Thus $(p / r)^{\prime}=p /(p-r)<\varepsilon_{G} /(r-2)$ and so $-\varepsilon_{G}<(2-r)(p / r)^{\prime}$.

By the Weyl integration formula, to show that $R$ is of type central $\Lambda(p, s)$, we have to show that there exists $K \in \mathbf{R}$ such that for all sequences $\left(a_{\sigma}\right)_{\sigma \in R}$ with $a_{\sigma}=0$ off a finite set,

$$
\left(\left.\int_{T}\left|\sum_{\sigma \in R} a_{\sigma} \chi_{\sigma}\right| T\right|^{r}|q|^{2} d \lambda_{T}\right)^{1 / r} \leq K\left(\sum_{\alpha \in R} d_{\sigma}^{2-s}\left|a_{\sigma}\right|^{2}\right)^{1 / s}
$$

Now, applying Hölder's inequality,

$$
\begin{gathered}
\left(\left.\int_{T}\left|\sum_{r \in R} a_{\sigma} \chi_{\sigma}\right| T\right|^{r}|q|^{2} d \lambda_{T}\right)^{1 / r}=\left(\left.\int_{T}\left|\sum_{\sigma \in R} a_{\sigma} q \cdot \chi_{\sigma}\right| T\right|^{r}|q|^{2-r} d \lambda_{T}\right)^{1 / r} \\
\leq\left(\int|q|^{(2-r)(p / r)^{\prime}} d \lambda_{T}\right)^{(p-r) / p}\left(\left.\int\left|\sum a_{\sigma} q \cdot \chi_{\sigma}\right| T\right|^{p} d^{p} \lambda_{T}^{1 / p}\right. \\
=K(p, r)\left(\int\left|\sum a_{\sigma} q \cdot \chi_{\sigma}\right| T \mid d^{p} \lambda_{T}\right)^{1 / p} .
\end{gathered}
$$

I have denoted by $K(p, r)$ the number $\left(\int|q|^{(2-r)(p / r)^{\prime}} d \lambda_{T}\right)^{(1 / r-1 / p)}$. 
By the Weyl character formula, and the elementary properties of the $p$-norm, we have

$$
\begin{aligned}
\left(\left.\int\left|\sum a_{\sigma} q \chi\right| T\right|^{p} d \lambda_{T}\right)^{1 / p} & \leq \sum_{w \in W}\left(\int\left|\sum a_{\sigma} w\left(\chi^{(\sigma)}\right) \bar{\chi}_{(w)}\right|^{p} d \lambda_{T}\right)^{1 / p} \\
& =\operatorname{card} W\left\|\sum_{\sigma \in R} a_{\sigma} \chi^{(\sigma)}\right\|_{p} .
\end{aligned}
$$

Thus, since $X(R)$ is of type $d^{2-s}-\Lambda(p, s)$, we have the required inequality.

(2.3) Of course for $r>2$ the sets of type $d^{0}-\Lambda(r, 2)$ are simply sets of type $\Lambda(r)$ (see [5]). Thus we obtain

COROLLARY. Suppose $G$ is a compact connected Lie group. Let $2<r<$ $2+\varepsilon_{G}$. Then in order for $R \subseteq \hat{G}$ to be of type central $\Lambda(r)$ it is sufficient that $X(R)$ should be of type $\Lambda(p)$ for some $p \in\left(\left(r \cdot \varepsilon_{G}\right) /\left(2+\varepsilon_{G}-r\right), \infty\right)$.

In particular, every infinite subset of $\hat{G}$ contains an infinite set which is of type central $\Lambda(r)$ for all $r<2+\varepsilon_{G}$.

(2.4) COROLLARY. Suppose $G$ is an infinite compact connected group. Then for some $p>2, \hat{G}$ contains an infinite central $\Lambda(p)$ set.

PROOF. By the well-known structure theorem, we may write $G=G_{1} \times G_{2}$ where $G_{1}$ is an infinite connected compact Lie group. Now $\hat{G}_{1}$ certainly contains an infinite central $\Lambda(p)$ set, $R$, and it is not hard to check that, considered as a subset of $\hat{G}, R$ is a central $\Lambda(p)$ set.

\section{Sets of type central $V(p, q)$}

(3.1) Definition. Let $T, w$ be as in (2.1). Suppose $p, s \in[1, \infty], s \neq 1$. Then $Y \subseteq \hat{T}$ is of type $w-V(p, s)$ if there is $K \in \mathbf{R}$ so that for all sequences $\left(a_{\chi}\right)_{X \in Y}$, zero off a finite set,

$$
\left(\sum_{\chi \in Y} w(\chi)\left|a_{\chi}\right|^{r ;}\right)^{1 / r^{\prime}} \leq K\left\|\sum_{\chi \in Y} a_{\chi} \chi\right\|_{p^{\prime}} .
$$

(3.2) Proposition. Let $p \in[2, \infty], r \in(1, \infty]$. If $R \subseteq \hat{G}$ is of type central $V(p, r)$, then

$$
X(R) \subseteq \hat{T}^{+} \text {is of type } d^{2-r^{\prime}}-V(p, r) .
$$


PrOOF. Let $\left(a_{\sigma}\right)_{\sigma \in R}$ be zero off a finite set. Then we must show that

$$
\left(\sum_{\sigma \in R} d_{\sigma}^{2-r^{\prime}}\left|a_{\sigma}\right|^{r^{\prime}}\right)^{1 / r^{\prime}} \leq K\left\|\sum_{\sigma \in R} a_{\sigma} \chi^{(\sigma)}\right\|_{p^{\prime}} .
$$

By the Weyl character formula and the Weyl integration formula,

$$
\begin{aligned}
\left\|\sum_{\sigma \in R} a_{\sigma} \chi^{(\sigma)}\right\|_{p^{\prime}} & \geq \operatorname{card} W^{-1^{\prime}}\left\|\sum_{\sigma \in R} a_{\sigma} \sum_{w \in W} w \chi^{(\sigma)} \cdot \bar{\chi}_{(w)}\right\|_{p^{\prime}} \\
& =\operatorname{card} W^{-1^{\prime}} \| \sum_{\sigma \in R} a_{\sigma} q \cdot \chi_{\sigma}\left\lceil T \|_{p^{\prime}}\right. \\
& =\operatorname{card} W^{-1^{\prime}}\left(\int \mid \sum_{\sigma \in R} a_{\sigma} \chi_{\sigma}\left\lceil\left. T\right|^{p^{\prime}}|q|^{p^{\prime}} d \lambda_{T}\right)^{1 / p^{\prime}}\right. \\
& \geq \operatorname{card} W^{-3+p^{\prime}}\left(\left.\left|\int \sum_{\sigma \in R} a_{\sigma} \chi_{\sigma}\right| T\right|^{p^{\prime}}|q|^{2} d \lambda_{T}\right)^{1 / p^{\prime}} \\
& =\operatorname{card} W^{-2}\left(\int\left|\sum_{\sigma \in R} a_{\sigma} \chi_{\sigma}\right|^{p^{\prime}} d \lambda_{G}\right)^{1 / p^{\prime}}
\end{aligned}
$$

where the penultimate step follows from the fact that $\|q\|_{\infty}=\operatorname{card} W$. Since $R$ is of type central $V(p, r)$, we now have, for some $K \in \mathbf{R}$, independent of $\left(a_{\sigma}\right)$,

$$
\left(\sum_{\sigma \in R} d_{\sigma}^{2-r^{\prime}}\left|a_{\sigma}\right|^{r^{\prime}}\right)^{1 / r^{\prime}} \leq K \operatorname{card} W^{-2}\left\|\sum a_{\sigma} \chi^{(\sigma)}\right\|_{p^{\prime}}
$$

as required.

(3.3) THEOREM. Let $G$ be a compact connected Lie group, $T$ a maximal torus for $G$. Suppose $p \in[2, \infty]$ and $r \in(1, \infty]$, and let $R \subseteq \hat{G}$. Then if $\bigcup_{w \in W} w(\chi(R)) \bar{\chi}_{(w)}$ is of type $d^{2-r^{\prime}}-V(p, r), R$ is of type central $V(s, r) s<$ $\left(\left(2+\varepsilon_{G}\right) /\left(p+\varepsilon_{G}\right)\right) p$.

Proof. Suppose that $X(R)$ is of type $d^{2-r^{\prime}}-V(p, r)$ with constant $K$, and choose $s<\left(\left(2+\varepsilon_{G}\right) /\left(p+\varepsilon_{G}\right)\right) p$. It then follows that $p^{\prime}<s^{\prime}$ and that 
$p((s-2) /(p-s))<\varepsilon_{G}$. Thus for any sequence $\left(a_{\sigma}\right)_{\sigma \in R}$ with $a_{\sigma}=0$ off a finite set, we have, using the same techniques as in the calculation of (2.2),

$$
\begin{aligned}
\left(\sum d_{\sigma}^{2-r^{\prime}}\left|a_{\sigma}\right|^{r^{\prime}}\right)^{1 / r^{\prime}} & \\
\leq & K \operatorname{card} W^{1 / r^{\prime}}\left(\int\left|\sum_{\sigma \in R} a_{\sigma} \sum_{w \in W} w \chi^{(\sigma)} \bar{\chi}_{(w)}\right|^{p^{\prime}} d \lambda_{T}\right)^{1 / p^{\prime}} \\
= & K \operatorname{card} W^{-1 / r^{\prime}}\left(\left.\int\left|\sum_{\sigma \in R} a_{\sigma} q \cdot \chi_{\sigma}\right| T\right|^{p^{\prime}} d \lambda_{T}\right)^{1 / p^{\prime}} \\
= & K \operatorname{card} W^{-1 / r^{\prime}}\left(\left.\int\left|\sum_{\sigma \in R} a_{\sigma} \chi_{\sigma}\right| T q^{2 / s^{\prime}}\right|^{p^{\prime}}|q|^{p^{\prime}\left(1-2 / s^{\prime}\right)} d \lambda_{T}\right)^{1 / p^{\prime}} \\
\leq & K \operatorname{card} W^{-1 / r^{\prime}}\left(\left.\int\left|\sum_{\sigma \in R} a_{\sigma} \chi_{\sigma}\right| T\right|^{s^{\prime}}|q|^{2} d \lambda_{T}\right)^{1 / s^{\prime}} \\
& \times\left(\int|q|^{-p((s-2) /(p-2))} d \lambda_{T}\right)^{s^{\prime} /\left(s^{\prime}-p^{\prime}\right)} \\
= & K \operatorname{card} W^{1 / s^{\prime}-1 / r^{\prime}} \kappa_{1}(p, s)\left\|\sum_{\sigma \in R} a_{\sigma} \chi_{\sigma}\right\|_{s^{\prime}}
\end{aligned}
$$

This shows that $R$ is of type central $V(s, r)$.

(3.4) Recalling that a set is of type central $V(p, 2)$ for some $p>2$ if and only if it is of type central $\Lambda(2)$ in the sense of [8], we have

COROLLARY. Let $G$ be a compact connected Lie group, $T$ a maximal torus for $G$. Then $R \subseteq \hat{G}$ is a central $\Lambda(2)$ set if and only if $X(R) \subseteq \hat{T}$ is a $\Lambda(2)$ set.

(3.5) Proposition. Let $G$ be a compact connected Lie group, and suppose $R \subseteq \hat{G}$. Then if $\sum_{\sigma \in R} d_{\sigma}^{-\varepsilon_{G}}<\infty, R$ is a set of type central $V(s, r)$ for all $(s, r)$ satisfying $r^{\prime} \leq 2$ and $2 \leq s<2+\varepsilon_{G}$.

ProOF. Since $2 \leq s<2+\varepsilon_{G}$, we may choose $p \in(2, \infty)$ so that $\max \left(r^{\prime}, s\right)<$ $\left(\left(2+\varepsilon_{G}\right) /\left(p+\varepsilon_{G}\right)\right)^{p}<2+\varepsilon_{G}$. Notice that $1<\varepsilon_{G} /\left(r^{\prime}-2\right)<\infty$. Thus, applying 
Hölder's inequality, we obtain, for any sequence $\left(a_{\sigma}\right)_{\sigma \in R}$

$$
\begin{aligned}
\left(\sum_{\sigma \in R} d_{\sigma}^{2-r^{\prime}}\left|a_{\sigma}\right|^{r^{\prime}}\right)^{1 / r^{\prime}} \leq & \left(\sum_{\sigma \in R} d_{\sigma}^{-\varepsilon_{G}}\right)^{\left(r^{\prime}-2\right) /\left(r^{\prime} \varepsilon_{G}\right)} \\
& \times\left(\sum_{\sigma \in R}\left|a_{\sigma}\right|^{r^{\prime} \varepsilon_{G} /\left(2+\varepsilon_{G}-r^{\prime}\right)}\right)^{\left(2+\varepsilon_{G}-r^{\prime}\right) / r^{\prime} \varepsilon_{G}} \\
& \leq K_{1}\left(\sum_{\sigma \in R}\left|a_{\sigma}\right|^{p}\right)^{1 / p}
\end{aligned}
$$

where $K_{1}=\left(\sum d^{-\varepsilon_{\sigma}} G\right)^{\left(r^{\prime}-2\right) / r^{\prime} \varepsilon_{G}}$. The second inequality follows once we note that $r^{\prime}<\left(\left(2+\varepsilon_{G}\right) /\left(p+\varepsilon_{G}\right)\right) p$ is equivalent to $r \varepsilon_{G} /\left(2+\varepsilon_{G}-r^{\prime}\right)<p$. Now by the Hausdorff-Young inequality

$$
\begin{aligned}
\left(\sum_{\sigma \in R}\left|a_{\sigma}\right|^{p}\right)^{1 / p} & \leq \operatorname{card} W^{-1 / p}\left\|\sum_{\sigma \in R} a_{\sigma} \sum_{w \in W} \operatorname{sgn} w \chi^{(\sigma)} \bar{\chi}_{(w)}\right\|_{p^{\prime}} \\
& =\operatorname{card} W^{-1 / p} \| \sum_{\sigma \in R} a_{\sigma} q \cdot \chi_{\sigma}\left\lceil T \|_{p^{\prime}} .\right.
\end{aligned}
$$

But as part of the proof of (3.3) it is shown that

$$
\| \sum a_{\sigma} q \cdot \chi_{\sigma}\left\lceil T\left\|_{p^{\prime}} \leq K_{2}\right\| \sum a_{\sigma} \chi_{\sigma} \|_{s^{\prime}}\right.
$$

whenever $s<\left(\left(2+\varepsilon_{G}\right) /\left(p+\varepsilon_{G}\right)\right) p$. This completes the proof.

(3.6) EXAMPLE. It is easily seen that a set of type central $\Lambda(2)$ is of type central $V(s, r)$ whenever $\inf \left(s, r^{\prime}\right)>2$. The converse, however, does not hold; there exist sets in $S U(2)^{\wedge}$ which are of type central $V(s, r)$ for all $s, r$ satisfying $\max \left(r, s^{\prime}\right)<3$ but which are not of type central $\Lambda(2)$. (By (3.4) and (3.5), it suffices to find a set $E$ of integers such that $\sum_{n \in E} n^{-1}<\infty$, but such that $E$ is not a $\Lambda(2)$ set-such a set is given by $E=\{2 m !+n(2 m) !: 0 \leq n \leq m, m \in N\}$. It is shown in $[13,(4.3)]$ that this set is not a $\Lambda(2)$ set.)

\section{Central $p$-Sidon sets}

(4.1) Sets of type central $V\left(1, p^{\prime}\right), 1 \leq p<2$, are called central $p$-Sidon sets. Central 1-Sidon sets are central Sidon sets in the sense of [8], [10]. In [3] it is shown that if $G$ is semisimple, then $\hat{G}$ contains no infinite $p$-Sidon sets. It is shown [10] for general compact connected $G$ that $\hat{G}$ contains an infinite central 
1-Sidon set if and only if $G$ is not semisimple Lie. The results of this section indicate that for central $p$-Sidon sets, $p>1$, the situation is radically different.

(4.2) Suppose $G$ is a compact connected semisimple Lie group, and suppose (see (1.3)) that $\tilde{\rho}$ is a weight of $G$. Then for each integer $n$, there is a representation $\sigma_{n} \in \hat{G}$ so that $\tilde{\chi}^{\left(\sigma_{n}\right)}=n \tilde{\rho}$. For general compact connected semisimple $G$, this will be true only for even $n$. I will show that if $\left(b_{n}\right)_{n \in \mathrm{N}}$ is a sufficiently lacunary sequence, then $\left\{\sigma_{b_{n}}\right\}$ is a central $p$-Sidon set, where if $\tilde{\rho}$ is not a weight of $G$, we understand that $\left(b_{n}\right)$ consists only of even integers.

(4.3) LEMMA. If $G$ is any compact connected semisimple group and $\sigma_{n}$ is as in (4.2), then

(i) $d \sigma_{n}=(n+1)^{\mathrm{card} \Phi^{+}}$.

(ii) $\chi_{\sigma_{n}}(z)=\prod_{\alpha \in \Phi^{+}} D_{n / 2}(\alpha(z))$ for all $z \in T$

where $D_{n}$ is the Dirichlet kernel,

$$
D_{n}(z)=\frac{z^{n+1 / 2}-\bar{z}^{(n+1 / 2)}}{z^{1 / 2}-\bar{z}^{1 / 2}} .
$$

Proof. (i) is a direct result of the Weyl dimension formula, and (ii) is true because by the Weyl character formula $\left.\chi \sigma_{n}\right|_{T}=\mathcal{A}\left(\rho^{n+1}\right) / \mathcal{A}(\rho)$. Now from (1.2), $A(\rho)=\prod_{\alpha \in \Phi^{+}}\left(\alpha^{1 / 2}-\bar{\alpha}^{1 / 2}\right)$, and from this it easily follows that $A\left(\rho^{n+1}\right)=\prod_{\alpha \in \Phi^{+}}\left(\alpha^{(n+1) / 2}-\bar{\alpha}^{(n+1) / 2}\right)$.

(4.4) Let $\beta_{1}$ be any element of $\Delta$ : set

$$
T_{1}=\left\{z \in T: \beta(z)=1 \text { for } \beta \in \Delta \backslash\left\{\beta_{1}\right\}\right\} .
$$

Since the elements of $\tilde{\Delta}$ form a basis for $C L(T)^{*}, T_{1}$ is a one-dimensional torus. Now for each $\alpha \in \Phi^{+}$, we can write $\alpha=\prod_{\beta \in \Delta} \beta^{m_{\beta}^{\alpha}}$, where the $m_{\beta}^{\alpha}$ s are nonnegative integers. Let $m_{1}^{\alpha}=m_{\beta_{1}}^{\alpha}$ and $\Phi_{1}^{+}=\left\{\alpha \in \Phi^{+}: m_{1}^{\alpha} \neq 0\right\}$. Then it is a direct result of (4.3)(ii) that for $z \in T_{1}$,

$$
\chi_{\sigma_{n}}(z)=(n+1)^{\operatorname{card}\left(\Phi^{+} \backslash \Phi_{1}^{+}\right)} \prod_{\alpha \in \Phi_{1}^{+}} D_{n / 2}\left(z^{m_{1}^{\alpha}}\right)
$$

(4.5) LEMMA. Let $G$ be a compact Lie group with maximul torus $T$, and let $R \subseteq \hat{G}$. Then $R$ is a central $p$-Sidon set if and only if there is a constant $K$ such that for all sequences $\left(a_{\sigma}\right)_{\sigma \in R}$ of complex numbers, zero off a finite set,

$$
\left(\sum_{\alpha \in R}\left|a_{\sigma}\right|^{p}\right)^{1 / p} \leq K \| \sum_{\sigma \in R} d_{\sigma}^{1-2 / p} a_{\sigma} \chi_{\sigma}\left\lceil T \|_{\infty} .\right.
$$


PrOOF. This lemma is a direct consequence of the definition, together with the fact that for $f$ central, $\| f\left\lceil T\|-\infty=\| f \|_{\infty}\right.$.

(4.6) In view of (4.4), it will come as no surprise to the reader that we need to examine functions which are products of Dirichlet kernels. The technical result which we requiure for Theorem (4.8) is contained in the following Proposition.

Proposition. Suppose $\left\{m_{1}=1, m_{2}, \ldots, m_{t}\right\}$ is a set of positive integers with sum $S$.

For each positive integer $c$, and for each $z \in T$, let $H_{c}(z)=\prod_{i=1}^{t} D_{c}\left(z^{m_{i}}\right)$.

Further, for each pair $0<a<b$ of positive integers, let

$$
F_{a, b}(z)=z^{1 / 2(a+b) S} F_{1 / 2(a-b) S}(z),
$$

where

$$
F_{n}(z)=\left(\left(z^{1 / 2(n+1)}-\bar{z}^{1 / 2(n+1)}\right) /\left(z^{1 / 2}-\bar{z}^{1 / 2}\right)\right)^{2} /(n+1)
$$

is the usual Fejér kernel. ( $F_{a, b}$ is simply the Fejér kernel translated and dilated so that its Fourier transform is supported on the interval $(S a, S b)$.)

Then

(i) If $a>c, F_{a, b} * H_{c}=0$.

(ii) If $b \leq c,\left|F_{a, b} * H_{c}(1)\right| \leq S(b-a)(2 c+1)^{t-1}$.

(iii)

$$
\left|F_{a, b} * H_{b}(1)\right| \geq \frac{2 R^{t+1}}{S\left(1+\frac{a}{b}\right)(t+1) !} \prod_{i=1}^{t} m_{i} \cdot b^{t}
$$

where

$$
R=\min \left(\frac{S}{2}\left(1-\frac{a}{b}\right), 1\right)
$$

ProOF. We denote $D_{c}\left(z^{m_{i}}\right)$ by $D_{c}^{i}(z)$. Then

$$
H_{c}^{\wedge}=\stackrel{t}{*} D_{c}^{i}
$$

where $*$ denotes convolution in $\mathbf{Z}$. Recall that

$$
D_{c}^{i \wedge}(n)= \begin{cases}1 & \text { if } n \in\left\{k \cdot m_{i}:|k| \leq c\right\}, \\ 0 & \text { otherwise. }\end{cases}
$$

From this, it follows that $H_{c}^{\wedge}(n)=0$ if $n \geq S \cdot c$, whence (i).

For (ii), observe that $F_{a, b}^{\wedge}$ and $H_{c}^{\wedge}$ are both positive functions: thus

$$
\left|F_{a, b} * H_{c}(1)\right|=\sum_{k \in \mathbf{Z}} F_{a, b}^{\wedge}(k) H_{c}^{\wedge}(k) .
$$


Moreover, by (1), for all $k, H_{c}^{\wedge}(k) \leq H_{c}^{\wedge}(0)$. Therefore

$$
\begin{aligned}
\left|F_{a, b} * H_{c}(1)\right| & \leq H_{c}^{\wedge}(0) \sum_{k \in \mathbf{Z}} F_{a, b}^{\wedge}(k) \\
& =H_{c}^{\wedge}(0) F_{a, b}(1) \\
& =H_{c}^{\wedge}(0) S(b-a) .
\end{aligned}
$$

Now

$$
\begin{aligned}
\left|H_{c}^{\wedge}(0)\right| & \leq\left\|H_{c}^{\wedge}\right\|_{\infty} \leq\left\|D_{c}^{1 \wedge}\right\|_{l^{\prime}}\left\|\begin{array}{l}
t \\
* \\
i=2
\end{array} D_{c}^{i \wedge}\right\|_{l^{\infty}} \\
& =(2 c+1) \| \underset{\left\|_{i=2}^{*} D_{c}^{i \wedge}\right\|_{l^{\infty}}}{ },
\end{aligned}
$$

so we obtain

$$
\left|H_{c}^{\wedge}(0)\right| \leq(2 c+1)^{t-1} .
$$

Combining (3) and (4) proves (ii).

We now prove (iii). It is an easy exercise in induction to prove that if $S b-b \leq$ $k \leq S b$, then

$$
H_{b}^{\wedge}(k)=Q(S b-k)
$$

where $Q$ is a polynomial of degree $t-1$;

$$
Q(x)=\sum_{i=0}^{t-1} q_{i} x^{i}, \quad \text { where } q_{t-1}=\frac{1}{t !}\left(\prod_{i=1}^{t} m_{i}\right)^{-1} .
$$

The proof of this identity, which is left to the reader, makes use of the fact that $\sum_{j=0}^{n} j^{p}$ is a polynomial in $n$ of degree $p+1$, with leading coefficient $1 /(p+1)$. Secondly, we remark that if $S(b+a) / 2 \leq k \leq S b$ then

$$
F_{a, b}^{\wedge}(k)=\frac{2}{S(b-a)}(S b-k) \text {. }
$$

Choose

$$
R=\min \left(\frac{S}{2}\left(1-\frac{a}{b}\right), 1\right)
$$


Then if $S b-R b \leq k \leq S b,(5)$ and (6) are satisfied by $k$. Therefore we have

$$
\begin{aligned}
\sum_{k} F_{a, b}^{\wedge}(k) H_{b}^{\wedge}(k) & \geq \sum_{S b-R b \leq k \leq S b} F_{a, b}^{\wedge}(k) H_{b}^{\wedge}(k) \\
& \geq \frac{2}{S(b-a)} \sum_{0 \leq k \leq R b} Q(k) \cdot k \\
& \geq \frac{2}{S(b-a)} q_{t-1} \sum_{0 \leq k \leq R b} k^{t} \\
& \geq \frac{2}{S(b-a)} q_{t-1} \frac{(R \cdot b)^{t+1}}{t+1} \\
& =2 R^{t+1}\left(\prod_{i=1}^{t} m_{i}\right)^{-1}\left(s\left(1-\frac{a}{b}\right)(t+1) !\right)^{-1} b^{t}
\end{aligned}
$$

This completes the proof.

(4.7) Notation. If $G$ is a compact connected Lie group, we denote by $\lambda_{G}$ the smallest $\lambda \geq 1$ satisfying the inequality

$$
2^{\operatorname{card} \Phi_{1}^{+}}\left(\operatorname{card} \Phi_{1}^{+}+1\right) ! \prod_{\alpha \in \Phi_{1}^{+}} m_{1}^{\alpha}\left\langle\tilde{\lambda}_{\beta_{1}}, \tilde{\rho}\right\rangle<\frac{\lambda\left(1-\frac{1}{\lambda}\right)^{\operatorname{card} \Phi_{1}^{+}}}{\left(1+\frac{1}{\lambda}\right)} .
$$

(4.8) THEOREM. Let $G$ be a compact connected semisimple Lie group. Let $\left(b_{n}\right)_{n \in N}$ be a Hadamard sequence of integers with $b_{n+1} / b_{n}>\lambda_{G}$. Then $R=$ $\left\{\sigma_{b_{n}}: n \in \mathbf{N}\right\}$ is a central $p$-Sidon set for every $p>1$.

ProOF. Let $\left(a_{\sigma}\right)_{\sigma \in R}$ be a sequence of complex numbers, zero off a finite set. For notational convenience, we let

$$
f=\sum_{\sigma \in R} d_{\sigma}^{1-2 / p} a_{\sigma} \chi_{\sigma}\left\lceil T_{1} .\right.
$$

Then

$$
\|f\|_{\infty} \leq \| \sum_{\sigma \in R} d_{\sigma}^{1-2 / p} a_{\sigma} \chi_{\sigma}\left\lceil T \|_{\infty}\right.
$$

By (4.3) and (4.4), for $z \in T_{1}$,

$$
f(z)=\sum_{n \in N}\left(b_{n}+1\right)^{2 \operatorname{card} \Phi^{+} / p^{\prime}-\operatorname{card} \Phi_{1}^{+}} a_{\sigma_{b_{n}}} H_{b_{n} / 2}(z) .
$$

$H_{b_{n} / 2}$ is defined as in Lemma (4.6), where we take $t=\operatorname{card} \Phi_{1}^{+}$, and the integers $m_{i}$ to be the integers $\left\{m_{1}^{\alpha} ; \alpha \in \Phi_{1}^{+}\right\}$of (4.4). 
To simplify the notation, I will denote $a_{\sigma_{b_{n}}}$ by $c_{n}, H_{b_{n} / 2}$ by $H_{n}^{\prime}$ and $F_{b_{n-1} / 2, b_{n} / 2}$ (also defined as in Lemma (4.6)) by $F_{n}^{\prime}$.

The basic idea of the proof is to show that for some $K_{2}>0$, and for all $n \in \mathbf{N}$,

$$
\left|c_{n}\right| \leq K_{2}\|f\|_{\infty}\left(b_{n}+1\right)^{-r}
$$

where $r=2 \operatorname{card} \Phi^{+} / p^{\prime}>0$.

Remark that for all $n \in \mathbf{N},\left\|F_{N}^{\prime}\right\|=1$. Therefore, we have

$$
\left|F_{N}^{\prime} * f(1)\right| \leq\left\|F_{N}^{\prime} * f\right\|_{\infty} \leq\|f\|_{\infty}
$$

and by the definition of $f$, we have

$$
F_{N}^{\prime} * f(1)=\sum_{n \in N}\left(b_{n}+1\right)^{r-t} c_{n} F_{N}^{\prime} * H_{n}^{\prime}(1)
$$

Now Lemma (4.6) gives us the following information.

(i) If $N>n$, then $F_{N}^{\prime} * H_{n}^{\prime}(1)=0$.

(ii) If $N \leq n$, then $\left|F_{N}^{\prime} * H_{n}^{\prime}(1)\right| \leq(S / 2)\left(b_{N}-b_{N-1}\right)\left(b_{n}+1\right)^{t-1}$.

(iii) $\left|F_{n}^{\prime} * H_{n}^{\prime}(1)\right| \geq K_{1} b_{n}^{t}$, where

$$
K_{1}=\left(2^{t}(t+1)^{\prime} \prod_{i=1}^{t} m_{i}\right)^{-1}\left(1-\frac{1}{\lambda}\right)^{t}\left(1+\frac{1}{\lambda}\right)^{-1} .
$$

Note that $S / 2=\frac{1}{2} \sum m_{1}^{\alpha}=\left\langle\tilde{\lambda}_{\beta_{1}}, \rho\right\rangle$, so $\lambda>\lambda_{G}$ guarantees that

$$
\left(1+\frac{2}{2 K_{1}}\right)<\lambda \text {. }
$$

Using the estimates (i), (ii) and (iii) on (10), we obtain

$$
\left|F_{N}^{\prime} * f(1)\right| \geq K_{1}\left(b_{N}+1\right)^{r}\left|c_{N}\right|-\frac{S}{2} \sum_{n>N}\left(b_{n}+1\right)^{r-1}\left(b_{N} * 1\right)\left|c_{n}\right| .
$$

Hence, by (9),

$$
\|f\|_{\infty}+\frac{S}{2}\left(b_{N}+1\right) \sum_{n>N}\left(b_{n}+1\right)^{r-1}\left|c_{n}\right| \geq K_{1}\left(b_{N}+1\right)^{r}\left|c_{N}\right| .
$$

Repeated application of (12) will yield (8). First, let $N_{0}$ be the largest integer with $c_{N_{0}} \neq 0$. We then have, by (12),

$$
K_{1}^{-1}\|f\|_{\infty} \geq\left(b_{N_{0}}+1\right)^{r}\left|c_{N_{0}}\right| .
$$

Combining (13) with the case $N=N_{1}-1$ of (8) gives

$$
\begin{gathered}
\left(b_{N_{0}-1}+1\right)^{r}\left|c_{N_{0}-1}\right| K_{1}^{-1}\left(\|f\|_{\infty}+\frac{S}{2} \frac{\left(b_{N_{0}-1}+1\right)}{\left(b_{N_{0}}+1\right)}\left(b_{N_{0}}+1\right)^{r}\left|c_{N_{0}}\right|\right) \\
\leq K_{1}^{-1}\left(1+\frac{S}{2 K_{1}} \cdot \lambda^{-1}\right)\|f\|_{\infty} .
\end{gathered}
$$


Continuing in this way, we obtain, for any $N<N_{0}$,

$$
\begin{aligned}
\left(b_{N}+1\right)^{r}\left|c_{N}\right| \leq & K_{1}^{-1}\left(1+\frac{S}{2 K_{1}} \lambda^{-1}+\frac{S}{2 K_{1}}\left(1+\frac{S}{2 K_{1}}\right) \lambda^{-2}+\cdots\right. \\
& \left.\cdots+\frac{S}{2 K_{1}}\left(1+\frac{S}{2 K_{1}}\right)^{N_{0}-N-1} \lambda^{N-N_{0}}\right)\|f\|_{\infty} \\
\leq & K_{1}^{-1}\left(1-\left(\left(1+\frac{S}{2 K_{1}}\right) \lambda^{-1}\right)^{N_{0}-N+1}\right) \\
& \times\left(1-\left(1+\frac{S}{2 K}\right) \lambda^{-1}\right)^{-1}\|f\|_{\infty} .
\end{aligned}
$$

We now invoke (11) to guarantee that the right-hand side of the above inequality is dominated by $K_{2}\|f\|_{\infty}$, where $K_{2}=K_{1}^{-1}\left(1-\left(1+S / 2 K_{1}\right) \lambda^{-1}\right)^{-1}$. Thus we have obtained (8). It follows that

$$
\left(\sum_{n \in N}\left|c_{n}\right|^{p}\right)^{1 / p} \leq K_{2}\left(\sum_{n \in N}\left(b_{n}+1\right)^{-2 p \operatorname{card} \Phi^{+} / p^{\prime}}\right)^{1 / p}\|f\|_{\infty} .
$$

Since $b_{n+1} / b_{n}>\lambda>1$, the series on the right is summable provided $p>1$, and we have, by (7)

$$
\left(\sum_{\sigma \in R}\left|a_{\sigma}\right|^{p}\right)^{1 / p} \leq K \| \sum_{\sigma \in R} d_{\sigma}^{-12 / p} a_{\sigma} \chi_{\sigma}\left\lceil T \|_{\infty} .\right.
$$

Thus, by Lemma (4.5), $R$ is central $p$-Sidon.

(4.9) REMARKS. (i) In general, the value for $\lambda_{G}$ given in (4.7) is not optimal. For $G=S U(2)$, the estimates of Lemma (4.6) become particularly simple, and I am able to show that Theorem (4.6) is valid with $\lambda_{G}=1$, but (4.7) would give $\lambda_{G}=3$. It would be interesting to know whether this improvement is possible in general.

(ii) It would be desirable to decide whether every infinite subset of $\hat{G}$ contains an infinite set which is central $p$-Sidon for some (or all) $p>1$. However, my proof of Theorem (4.8) depends critically on the fact that $\chi_{\sigma_{n}} \mid T_{1}$ can be factorized in a particularly simple way; such a factorization is not possible for $\chi_{\sigma} \mid T_{1}, \sigma$ arbitrary, except in special cases (cf. 4.11).

(4.10) In the case where $G$ is a compact connected group which is not semisimple Lie, it is known [10] that $\hat{G}$ contains an infinite central Sidon set, which is, of course, central $p$-Sidon for all $p>1$. 
Thus we have

THEOREM. Let $G$ be a compact connected group. Then $\hat{G}$ contains an infinite set which is central $p$-Sidon for all $p>1$.

(4.11) For the case $G=S U(2)$ (resp. $S O(3)$ ), the set $\left\{\sigma_{n}: n \in \mathbf{N}\right\}$ (resp. $\left.\left\{\sigma_{2 n}: n \in \mathbf{N}\right\}\right)$ is the entire dual of $G$. Thus we have

COROLLARY. Let $G=S U(2)$ or $S O(3)$. Then every infinite subset of $\hat{G}$ contains an infinite set which is central $p$-Sidon for all $p>1$.

\section{References}

[1] R. Blei, 'Combinatorial dimension and certain norms in harmonic analysis', Amer. J. Math., to appear.

[2] D. Cartwright and J. R. McMullen, 'A structural criterion for the existence of infinite Sidon sets', Pacific J. Math. 96 (1981), 301-317.

[3] A. H. Dooley, 'On lacunary sets for nonabelian groups', J. Austral. Math. Soc. Ser. A 25 (1978), 167-175.

[4] A. H. Dooley and P. M. Soardi, 'Local p-Sidon sets for Lie groups', Proc. Amer. Math. Soc. 72 (1978), 125-126.

[5] R. E. Edwards and K. A. Ross, 'p-Sidon sets', J. Functional Anal. 15 (1974), 404-427.

[6] J. E. Humphreys, Introduction to Lie algebras and representation theory (Springer-Verlag, Berlin, 1972).

[7] G. Johnson and G. S. Woodward, 'On p-Sidon sets', Indiana Univ. Math. J. 24 (1974/75), 161-167.

[8] W. A. Parker, 'Central Sidon and central $\Lambda_{p}$ sets', J. Austral. Math. Soc. 14 (1972), 62-74.

[9] J. F. Price, 'Non ci sono insiemi di tipo $\Lambda_{p}$ per $S U(2)$ ', Boll. Un. Mat. Ital. (4) 4 (1971), 871-881.

[10] D. Rider, 'Central lacunary sets', Monatsh. Math. 76 (1972), 328-338.

[11] _ , 'Norms of characters and central $\Lambda(p)$ sets for $U(n)$ ', (Lecture Notes in Mathematics 266, pp. 287-294, Springer-Verlag, Berlin and New York, 1972).

[12], , ' $S U(n)$ has no infinite local $\Lambda_{p}$ sets', preprint.

[13] W. Rudin, 'Trigonometric series with gaps', J. Math. Mech. 9 (1960), 203-227.

[14] J. W. Sanders, 'Weighted Sidon sets', Pacific J. Math. 63 (1976), 225-279.

[15] N. R. Wallach, Harmonic analysis on homogeneous spaces (Marcel Dekker, New York, 1973).

School of Mathematics

University of New South Wales

P.O. Box 1, Kensington, N.S.W. 2033

Australia 